\title{
Ciencia Para Todos: Guías Para Superar los Desafíos de la Comunicación Científica en Casos de Comunicación de la SALUD
}

\section{Science for All: Guides to Overcome the Challenges of Science Communication in Cases of Health Communication}

\section{Denisse VÁSQUEZ-GUEVARA}

MCM, PH.D. Universidad de Cuenca, Profesora Titular, Carrera de Comunicación, Cuenca-Ecuador Ph.D. Fellow Researcher, Latin American \& Iberian Institute, University of New Mexico

Albuquerque, New Mexico, EEUU

E-mail: denisse.vasquez@ucuenca.edu.ec

(iD) https://orcid.org/0000-0003-2703-1416

Fecha de recepción del artículo: 31/12/2019

Fecha de aceptación definitiva: 30/03/2020

\section{RESUMEN}

Este estudio explora los modelos de comunicación científica del déficit y el diálogo y sus resultados en investigaciones previas. El marco teórico presentado nutre a la metodología del estudio con las sugerencias de estudios de investigación-acción participativa en la investigación y práctica de la comunicación científica. En consecuencia, este estudio aplicó las metodologías de investigación cualitativa e investigación-acción participativa, con fundamentos de epistemología decolonial. Posteriormente, se aplicó el co-diseño de estrategias comunicacionales en dos programas de salud, uno para niños (Estados Unidos), y otro para adolescentes (Ecuador). Para el análisis de datos se combina la teoría de los dos pasos, teoría del framing y teoría del médium. El co-diseño participativo contribuyó en: a) facilitar el diálogo entre investigadores y sus audiencias; y b) crear estrategias comunicacionales adaptadas a las audiencias para proponer guías de comunicación científica para la salud. Los resultados evidencian la importancia del involucramiento de los investigadores como voceros, algunos criterios de diseño de mensajes para noexpertos, y criterios para seleccionar canales comunicacionales.

Palabras clave: Comunicación Científica; Comunicación de la Salud; Comunicación Estratégica.

\begin{abstract}
This study explores the science communication models of deficit and dialogue and their results in prior research. The theoretical framework presented in this study informs the overall study's methodology with suggestions for participatory action research studies in the practice and research of science communication. Consequently, this study applied qualitative research and participatory action research methodologies, along with
\end{abstract}


decolonial epistemological foundations. Consequently, the co-design of science communication strategies was applied in two health programs, one for children (United States), and another for teenagers (Ecuador). For data analysis, the study used the following theories: two-step theory, framing theory and medium theory. These were combined to interpret the data. The overall participatory co-design contributed to: a) facilitate the dialogue between researchers and their audiences; and b) create communication strategies adapted to audiences to propose scientific communication guides for health. The results demonstrate the importance of the involvement of researchers as spokespersons of their studies; several criteria for the design of messages for non-experts; and communication media selection criterion.

Keywords: Science Communication, Health Communication, Strategic Communication.

\section{Introducción y situación actual a tratar}

\subsection{Comunicación científica: una disciplina en evolución}

La tarea de definir la comunicación científica representa un desafío reconocido por varios autores debido a la constante evolución de la investigación de esta disciplina. Es así que la comunicación científica cambia dependiendo directamente acorde a los distintos objetivos que dirigieron a cada una de las corrientes de investigación. Es así como la comunicación científica se la conoce también como divulgación científica, cultura científica, la educación informal de la ciencia (Rocha, Massarani, Pedersoli, 2017).

De igual manera, en la comunicación científica, diversas corrientes de investigación -influidas por el contexto histórico- esta disciplina ha sido denominada también como: alfabetismo científico (Gregory \& Miller, 1998), entendimiento público de la ciencia, mejor conocido como Public Understanding of Science o PUS (por sus siglas en inglés) (Stilgoe \& Wilsdon, 2014), y más recientemente, en las últimas décadas se la denomina como comunicación científica para el interés e involucramiento público (Bowater \& Yeoman, 2013).

Las corrientes investigativas de la alfabetización científica, divulgación científica, cultura científica, la educación informal de la ciencia, hasta el entendimiento público de la ciencia (PUS), se fundamentaban en el modelo del déficit (Gregory \& Miller, 1998). El déficit asume que: (a) todo el conocimiento y experiencia de la ciencia está únicamente en la comunidad científica; (b) que a mayor conocimiento científico que se exponga a la sociedad se motivará a la apreciación de la ciencia; (c) el punto de partida para la comunicación de la ciencia debe asumir que las audiencias no poseen conocimiento y competencias para comprender la ciencia, de manera que son considerados como ignorantes (Bowater \& Yeoman, 2013).

Algunos modelos y teorías que contribuyeron al modelo del déficit son la teoría de la difusión de las innovaciones (Rogers, 2010). La difusión de las innovaciones, nace en Estados Unidos en la década de 1960 con el objetivo de explicar las etapas del proceso en el cual las innovaciones pasan/transitan para ser adoptadas por los grupos humanos. Esta teoría se fundamenta en una ideología capitalista que se fundamenta en la comunicación lineal y su poder de convencer a las audiencias, sin oportunidad al diálogo, desde el modelo de la aguja hipodérmica (Arroyave, 2006). Dicha teoría ha sido implementada en sociedades occidentales-hegemónicas para implementar cambios tecnológicos en la sociedad (Dupagne, 1999; Dutton, Rogers, \& Jun, 1987; 
Lin, 1998), cambios en salas de redacción en medios de comunicación (Abbot, Corbin, \& Neigergall, 2000; Garrison, 2000); y cambio organizacional en las empresas (Crawford \& Strohkich, 2002; James, Wotring, \& Forrest, 1995). Sin embargo, en América Latina, es rechazada y criticada por haber sido utilizada para las agendas de desarrollo hegemónico y aculturación (Beltrán 1976, Bordenave, 1976), que categorizan y enmarcan a América Latina en comparación a naciones occidentales como "tercer mundo, o una región subdesarrollada" (Martin-Barbero, 1993).

Como resultado, por medio del modelo del déficit, las actividades y estrategias de comunicación científica se desarrollaban con un enfoque informativo-educativo, por medio de procesos de comunicación unilateral y difusionista, que no permitían ni permiten el intercambio entre los científicos y la sociedad. Consecuentemente, la comunicación científica bajo estos parámetros falló en establecer relaciones de intercambio entre la ciencia y la sociedad (Stilgoe \& Wilsdon, 2008).

En la década de 1990, científicos y el gobierno del Reino Unido, por medio de la Royal Society ${ }^{\mathbf{1}}$ propuso y debatió sobre la necesidad de promover el diálogo entre la comunidad científica y la sociedad, y dejar a un lado los esquemas del déficit (Holliman et al. 2009). Esta discusión da lugar a la última corriente científica que se encuentra vigente hasta la actualidad: el modelo dialógico de comunicación científica.

La investigación se centró desde entonces en superar las limitaciones que no permiten el intercambio entre científicos y la sociedad, además de descubrir las problemáticas existentes a causa del modelo del déficit. Entre las principales problemáticas encontramos: el escepticismo de la sociedad hacia la ciencia y la falta de disponibilidad abierta de información científica. Además, en varios lugares del mundo la sociedad solicitó a la comunidad científica que sus voces sean escuchadas. Otro requerimiento es que científicos y la sociedad civil unan fuerzas para colaborar en torno a concientizar a los gobiernos y a sus legislaturas para cambiar leyes sobre problemas que aquejan a diversos grupos humanos (Wynne, 1995).

\subsection{Comunicación científica: del déficit al diálogo}

Desde entonces, dentro de la corriente investigativa del modelo del diálogo, el desafío se ha centrado en explorar en cómo promover la atención y el interés de la sociedad en la ciencia (Jensen \& Holliman, 2009) por medio de estrategias, actividades que motiven a la sociedad acercarse a la academia y a los centros de investigación.

De estas iniciativas nacen las sugerencias metodológicas y de aplicación estratégica para iniciativas de investigación en comunicación científica. Los fundamentos de Glaser y Strauss (1967) de la teoría de campo (grounded theory) fueron empleadas por los investigadores de comunicación científica para explorar las experiencias cotidianas de la sociedad como oportunidades para la comunicación científica.

Actualmente, la investigación de esta disciplina se centra principalmente, en la exploración de métodos que faciliten el intercambio y diálogo entre la ciencia y la sociedad; el desarrollo iniciativas de ciencia ciudadana (Brossard, Lewenstein \& Booney, 2005; Cooper, 2016); la comunicación científica en nuevos medios 2.0 y sus

1. The Royal Society es la organización independiente de científicos más antigua del mundo. Fue fundada en Londres, Reino Unido en 1660. Actualmente, promueve la excelencia en investigación científica alrededor del mundo y la comunicación de la ciencia. www.royalsociety.org 
efectos (Brossard, \& Scheufele, 2013; Lee \& VanDyke, 2018); además del desarrollo del periodismo científico (Dunwoody, 2014). Con estos cambios, el concepto de comunicación científica ha evolucionado hacia una nueva dirección.

Sin embargo, la comunicación científica aún requiere explorar cómo generar estrategias que permitan facilitar el diálogo y una mayor participación entre científicos y la sociedad. Por este motivo, el siguiente estudio se centró en co-diseñar diversas estrategias de comunicación científica por medio de dos programas de salud preventiva basados en investigación. Eat Smart to Play Hard (ESPH) de Health Sciences en University of New México, que atiende a niños y niñas de primaria del estado de Nuevo México, en Estados Unidos. ESPH promueve la alimentación saludable, y la actividad física por medio de un programa de seis semanas donde los niños(as) con el apoyo de sus familias y maestros aprenden a preparar recetas saludables y juegos de actividad física. Este programa se fundamenta en el modelo del mercadeo social (Grier \& Bryant, 2005; Wong et al., 2010). Por otra parte, ACTIVITAL, un programa desarrollado por el Departamento de Investigación de Biociencias de la Universidad de Cuenca, en Ecuador. ACTIVITAL atendió a niños, niñas de primaria y adolescentes y secundaria de la ciudad de Cuenca. Sus intervenciones se fundamentan en el modelo socio-cognitivo y socio-ecológico, para diseñar e implementar varias intervenciones como capacitaciones, juegos, recetarios, y monitoreo médico (Andrade et al., 2014).

\section{Objetivos}

Los objetivos fueron de este estudio fueron:

1) Construir guías metodológicas para investigadores y comunicadores estratégicos que trabajan con audiencias culturalmente diversas en comunicación de la salud.

2) Motivar el interés de las audiencias con espacios participativos con investigadores para mejorar la comunicación de programas de salud.

\section{Marco Teórico}

\subsection{Definiciones contemporáneas de la comunicación científica}

Producto del cambio de los modelos para la comunicación científica, a diferencia del déficit, los modelos dialógicos promueven que la comunicación ya no solamente se centre en los científicos o la academia, sino involucra a más actores que participan de forma activa en el proceso comunicacional. Weigold (2001). Entonces, se incluyen también como actores del proceso comunicativo de la ciencia a la audiencia meta, los científicos, los comunicadores estratégicos, los medios masivos tradicionales y periodistas como canales de comunicación.

De esta forma, se define a la comunicación científica como el uso de habilidades, actividades mediáticas masivas, y otras estrategias de diálogo para producir los siguientes efectos en las audiencias: atención hacia temas científicos y de innovación; disfrute y entretenimiento de la ciencia; interés evidenciado en el involucramiento voluntario de las audiencias con temas científicos; y el entendimiento del contenido científico, sus procesos y factores sociales de influencia (Bowater \& Yeoman, 2013). 


\subsection{Dificultades en la investigación de la comunicación científica}

Ante el cambio del modelo-paradigma para la investigación de la comunicación científica, la práctica más común de los investigadores/científicos interesados en difundir su investigación a la sociedad, fue buscar apoyo en las oficinas de comunicación o marketing de sus instituciones u universidades, y delegar este trabajo a los comunicadores estratégicos y/o buscar a los medios de comunicación para comunicar sus hallazgos (Bucchi, 2008). De esta manera, la responsabilidad del contenido y las estrategias recae sobre periodistas y comunicadores, mas no en los mismos investigadores (Friedman, Dunwoody \& Rogers, 1999). Sin embargo, en estos escenarios, el contenido científico se veía afectado, muchas veces por la mala interpretación de hallazgos, y la inexactitud del contenido en notas de prensa y piezas comunicacionales, que como consecuencia distorsiona los verdaderos hallazgos de una investigación científica (Bucchi, 2008). Además, mediante prácticas tradicionales de comunicación estratégica y periodística, se reproducen prácticas propias del déficit, como la difusión unidireccional de contenidos, sin oportunidad de diálogo e intercambio (Bucchi \& Trench, 2008).

Por este motivo, se hizo necesario, la necesidad de investigar y reflexionar en nuevos esquemas para la comunicación científica, que permitan superar las dificultades y retos existentes. Algunos estudios sugirieron que se requería una nueva metodología, que involucre a todos los actores activamente en el proceso de planificación y desarrollo de la comunicación científica, para esto los métodos de investigación-acción participativa fueron los más recomendados por distintos autores (Bucchi, 2008; Holliman et al., 2009).

Es por esto que este estudio se desarrolló por medio de la investigación acción participativa, en busca de generar nuevas guías para investigadores y comunicadores estratégicos para comunicar sus proyectos a audiencias culturalmente diversas en Estados Unidos y Ecuador, para motivar su interés y participación en torno a programas de salud para niños y adolescentes.

Las preguntas de investigación fueron las siguientes:

P.I. 1. ¿Cómo los investigadores pueden desarrollar mejores estrategias de comunicación desde la perspectiva de sus audiencias?

P.I.2. ¿Cómo se puede mejorar el interés e involucramiento de las audiencias de comunicación científica?

\section{Metodología}

Esta investigación se desarrolló por medio un diseño metodológico que combinó los métodos de acción-participativa (Chevalier \& Buckles, 2013) e investigación cualitativa (Marshall \& Rossman, 2013), de manera que se ofrezca un marco exploratorio e inclusivo para el diálogo entre investigadores y sus audiencias meta, y de esta forma construir guías y consideraciones estratégicas para la planificación y ejecución de estrategias de comunicación científica. El presente estudio se desarrolla por medio casos múltiples de estudio (Yin, 2017) que permitió contrastar los resultados en distintos contextos culturales, pero en una misma temática (Flyvbjerg, 2001), en este caso la comunicación científica de programas de salud. De igual manera, los resultados fueron analizados y contrastados entre ambos casos de estudio, en sus unidades de análisis para asegurar la rigurosidad científica (Herriott \& Firestone, 1983). La primera 
unidad de análisis (u.a.1.), correspondiente a la P.I.1, es la comunicación de la ciencia de forma eficiente. La segunda unidad de análisis (u.a.2.), correspondiente a la P.I.2. es el mejoramiento del interés y el involucramiento de las audiencias meta de los proyectos de investigación.

\subsection{Casos de estudio}

\subsubsection{Eat Smart to Play Hard. Albuquerque, New Mexico, EEUU}

Eat Smart to Play Hard (ESTPH) es un programa de salud preventiva para niños desarrollado por el Centro de Investigación de Prevención de la University of New Mexico. Su objetivo principal es promover y educar a niños de educación primaria sobre la importancia de la nutrición saludable y la actividad física. Para este estudio, los participantes fueron los estudiantes de secundaria de Health Leadership High School (HLHS), (10 participantes) en el año 2018 que intervinieron en un proyecto piloto de jóvenes educadores de salud para niños y el equipo de investigadores de ESTPH ( 5 investigadores). Los participantes que formaron parte El HLHS noveno y décimo los alumnos de grado fueron capacitados como educadores de salud para niños de primaria en la Escuela Primaria Cien Aguas en Albuquerque como parte de su plan de estudios escolar. Por su participación fueron compensados con USD \$25.00 (dólares americanos).

\subsubsection{ACTIVITAL. Cuenca, Ecuador}

ACTIVITAL, es un programa de investigación nutrición saludable y actividad física para niños en edad Escolar. El mismo está a cargo del Grupo de Investigación de Nutrición y Actividad Física del Departamento de Biociencias de la Universidad de Cuenca. Para este estudio, los estudiantes colaboradores fueron los ex-participantes de ACTIVITAL en Cuenca, Ecuador (10 participantes) y los investigadores del programa (2 investigadoras). Los ex-participantes estuvieron expuestos a los mensajes del programa en el pasado como público objetivo y siguieron las actividades del programa. Posteriormente aportaron sus opiniones de sus experiencias vividas sobre cómo ACTIVITAL se puede comunicar de una manera más atractiva a otros adolescentes y a sus familias. En ambos casos, los participantes fueron reclutados de forma voluntaria por medio de las bases de datos de ambos programas y proporcionaron consentimiento informado y autorización de sus padres al ser menores de edad. Su identidad fue resguardada en todo el proceso de investigación. Adicionalmente, los participantes recibieron una compensación económica por su participación de USD \$25.00 (dólares americanos). Todos los procedimientos para este estudio fueron revisados y autorizados por el comité de bioética de UNM, UNM-IRB.

\subsection{Recolección de datos y análisis}

Para la recolección de datos se elaboraron cuatro talleres participativos, donde se aplicaron herramientas de recolección de datos, para facilitar la discusión entre los investigadores y sus audiencias meta. Para conducir el diálogo participativo en los talleres se empleó el proceso cíclico de acción-reflexión de McNiff (2014). Por medio de este procedimiento de 5 pasos el diálogo se centraba en: 1) identificar los 
problemas y barreras existentes; 2) describir dichos problemas y analizar las posibles causas; 3) Proponer alternativas para solucionar los problemas existentes; 4) Evaluar los posibles factores de influencia de implementación; y 5) proponer acciones o guías que mejoren el interés y la participación de las audiencias (Ver Figura 1).

Figura 1. Ciclos del proceso de acción-reflexión aplicados al estudio.

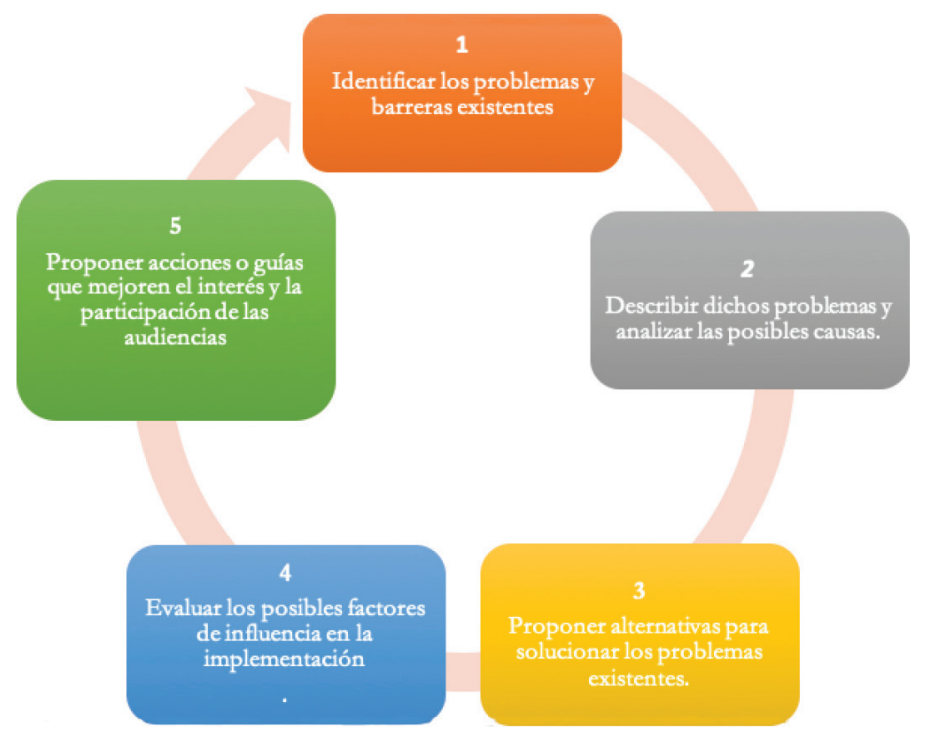

Fuente: Adaptación a este estudio de los ciclos de acción-reflexión (McNiff, 2014).

Las herramientas de recolección de datos empleadas fueron los diagramas participativos (Kesby, 2000), el mapeo de recursos (Chapin \& Threlkeld, 2001) y el fanzine (Guzzetti \& Gamboa, 2004). Cada taller fue grabado en audio. Luego de cada uno de los talleres, las grabaciones fueron transcritas y posteriormente codificadas. El proceso de análisis de datos fue colaborativo entre los investigadores y los participantes en su totalidad. Como estrategia de análisis de datos, se empleó la codificación abierta (Marshall \& Rossman, 2014). Los resultados emergentes se organizaron en categorías y luego en temáticas que se relacionaban con las unidades de análisis y su respectiva pregunta de investigación. Posteriormente los resultados fueron organizados y discutidos en contraste a tres teorías de la comunicación. La teoría de los dos pasos (Katz, 1957), para identificar los voceros y líderes de opinión ideales del contenido científico. La teoría del framing (Entman, 1993; Listerman, 2010; Gamson, 1992) para la discusión de parámetros clave para enfocar el diseño de los mensajes/contenido científico de forma que motive el interés de las audiencias. Finalmente, la teoría del médium (Meyrowitz, 2009), permitió la discusión y determinación de parámetros para la selección de canales comunicacionales para cada estrategia de comunicación científica.

\section{Resultados}

La siguiente sección presentará los resultados referentes a cada pregunta de investigación con su correspondiente unidad de análisis. Es fundamental recalcar que los 
participantes de cada caso de estudio respondieron en base a sus experiencias en relación a cada uno de los programas a los que pertenecían.

\section{1. ¿Cómo los investigadores pueden desarrollar mejores estrategias de comunicación desde la perspectiva de sus audiencias?}

Esta pregunta se resolvió mediante el primer y el segundo taller participativo y corresponde a la primera unidad de análisis (u.a.1.) comunicación de la ciencia de forma eficiente. En este aspecto se revisaron las estrategias de comunicación de cada programa, sus piezas comunicacionales, los mensajes y los canales empleados.

En ambos casos, los participantes de ambos casos de estudio, reconocieron que la información y recomendaciones de los programas eran importantes en cuanto a su contenido, y principalmente porque estaban elaboradas por científicos que son expertos en la salud.

Sin embargo, tanto los participantes de ESTPH y ACTIVITAL, mencionaron que desearían poder una comunicación directa con los investigadores para aprender e interactuar de forma directa con ellos. Los canales online, como redes sociales y las actividades offline, como eventos informales y conversatorios fueron sugeridos como alternativa. De esta manera, las audiencias meta afirmaron, que para ellos es importante no sólo recibir la información, sino poder interactuar con los investigadores de forma informal para aclarar sus dudas o poder hacer preguntas sobre su salud nutricional y la actividad física.

En el caso de los participantes de ESTPH, en Estados Unidos, un hallazgo diferenciador fue que los participantes sugirieron incorporar recursos que les apoyen a incorporar hábitos saludables. En específico, el acceso a frutas, vegetales y comida saludable que se ajuste al presupuesto de sus familias, o que les pueda ser obsequiado como parte de su participación en el programa.

De la discusión con los investigadores nació la iniciativa de implementar un espacio para jardines y siembra de vegetales en las escuelas con la colaboración de maestros y estudiantes para facilitar el acceso a comida saludable. Este hallazgo, no fue común en el caso de estudio en Ecuador, donde los participantes manifestaron que pueden acceder con facilidad a opciones saludables sin importar el presupuesto familiar.

\section{2. ¿Cómo se puede mejorar el interés e involucramiento de las audiencias de comunicación científica?}

La siguiente sección de resultados comprende a la segunda unidad de análisis (u.a.2.), es el mejoramiento del interés y el involucramiento de las audiencias meta de los proyectos de investigación, correspondiente a la P.I.2. Esta pregunta de investigación y su unidad de análisis se respondieron mediante el tercer y el cuarto taller participativo.

En cuanto a los canales adecuados para la comunicación científica para despertar el interés de las audiencias, ambos casos de estudio coincidieron en sugerir a las redes sociales, principalmente Instagram, Facebook y YouTube. De igual manera, en cuanto a contenido, los participantes del estudio supieron sugerir que debe ofrecerse 
contenido útil para mantener una alimentación saludable -como recetas de cocina, porciones adecuadas de alimentos y refrigerios saludables- y opciones de actividad física -como rutinas de baile y ejercicios que se puedan hacer en casa. Adicionalmente, en cuanto al uso de redes sociales, los participantes de ambos casos de estudio coincidieron con que una estrategia para motivar el interés y la atención de futuras audiencias sería implementar concursos en línea para las audiencias en general mediante el posteo de fotografías acompañadas de hashtags que registren el proceso de adoptar hábitos saludables de actividad física y de recetas saludables, y que premien vía sorteo o según el número de interacciones que generen los usuarios, con premios relacionados con la vida saludable. Por ejemplo, un día de senderismo y/o tarjetas de regalo de comida saludable.

Sin embargo, en el caso de Ecuador, los participantes sugirieron adicionalmente el uso de aplicaciones móviles, para motivar por medio del juego en dispositivos a niños y jóvenes a realizar actividad física y llevar un registro digital de su peso e ingesta de alimentos de acuerdo a su edad y estatura.

Otro hallazgo, específico a el caso de estudio de ACTIVITAL en Ecuador, fue que complementariamente a las estrategias de comunicación digital -mediante redes sociales y apps- las audiencias y los investigadores coincidieron en que tácticas adicionales para motivar el interés público son los eventos informales. Por ejemplo, organizar eventos en espacios públicos como parques mediante rutinas de ejercicios, y charlas o conversatorios de preguntas y respuestas con los investigadores expertos.

En cuanto al diseño de mensajes, participantes de ambos casos de estudio coincidieron en sus discusiones en los criterios de comunicación de resultados científicos sobre hábitos saludables. En específico, sugirieron que es necesario buscar un enfoque en las situaciones de la vida cotidiana, con las cuales las audiencias puedan identificarse de forma positiva. Por ejemplo, el momento del recreo en las escuelas, los juegos entre amigos, cocinar en casa, y los espacios de esparcimiento familiar como espacios de experiencias divertidas. De igual manera, mediante las discusiones de ambos casos de estudio, se concluyó que el lenguaje debe ser coloquial, libre de tecnicismos, o en su efecto proporcionar las explicaciones de los expertos para comprender a cabalidad conceptos, hallazgos o cualquier tipo de dato científico.

\section{Discusión de resultados}

Luego de revisar los principales hallazgos de este estudio, es evidente desde la perspectiva de las audiencias que existe la necesidad de implementar estrategias que promuevan la interacción y el diálogo entre investigadores y sus audiencias meta. En ambos casos de estudio se evidencia que las audiencias valoran el contenido y estrategias de ambos programas para promover hábitos saludables, también se corroboran los hallazgos de estudios previos. Dichos estudios argumentan sobre la necesidad de implementar espacios de diálogo directo e interacción de investigadores y sus audiencias para tratar temas que afectan a la sociedad y explorar posibles soluciones de aplicación (Servaes \& Malikhao, 2012; Wynne, 1995).

Sin embargo, de cierta manera, la comunicación científica aún es una tarea que se desarrolla por medio de esquemas de comunicación unidireccional, siguiendo esquemas del modelo del déficit, donde el investigador divulga información de su trabajo, pero no existen espacios o estrategias que promuevan el diálogo e intercambio 
(Bowater \& Yeoman, 2013). De esta manera, las iniciativas para motivar el interés público en la investigación científica aún son pocas.

Este estudio al ser realizado mediante una metodología participativa-cualitativa, permitió explorar de mejor manera, qué aspectos son fundamentales para mejorar el interés e intercambio entre científicos y sus audiencias. Como se evidencia en los resultados, se pudieron identificar elementos clave en cuanto a líderes de opinión, enfoque, diseño del mensaje, selección de canales y formatos adecuados para la comunicación científica. Sin embargo, vale aclarar que se limita a casos de estudio en la salud preventiva de niños, y si bien se brinda guías genéricas no se podría asegurar que dichas guías se apliquen a otros escenarios de estudios en otras disciplinas.

\section{Conclusiones}

El presente estudio permitió elaborar las siguientes guías para la comunicación científica para motivar el interés de las audiencias meta de niños, niñas y adolescentes. A continuación, el detalle de cada una de las guías resultantes de este estudio.

\subsection{Construir confianza y espacios de diálogo y experiencias con las audiencias}

Para implementar iniciativas o estrategias de comunicación es importante como primer paso, establecer confianza con las audiencias. En el caso de Estados Unidos y Ecuador, explorar la apertura de las audiencias de ambos proyectos permitió corroborar los principios de la teoría de los dos pasos (Katz, 1957). La teoría de los dos pasos establece que las credenciales académicas y de educación de los investigadores fueron valoradas por las audiencias, de manera que considerar la información que provenga de estudios elaborados por estos profesionales es creíble y de alto valor para las audiencias. Se afirma entonces lo que Katz expone al referirse a las credenciales científicas y formación profesional de los investigadores, los convierten en líderes de opinión ideales para ser los voceros de su propio trabajo científico y con alta credibilidad desde los ojos de las audiencias (1957).

En el caso de ambos estudios, las audiencias demostraron apertura e interés de trabajar y dialogar con investigadores. En opinión de los participantes, que son audiencias de cada programa de salud, coincidieron que tienen apertura, voluntad, e interés y sienten que la información que se les entregue de forma directa será confiable. En resumen, para las audiencias es interesante interactuar en diálogo directo con un investigador, siempre y cuando puedan participar de forma equitativa y recibir respuestas a sus inquietudes mediante eventos informales abiertos (conversatorios en espacios informales, como cafeterías, parques), o actividades online (por ejemplo, transmisiones en vivo o en redes sociales), será una actividad atractiva y confiable. Dichos eventos, deben también romper el esquema tradicional de divulgación científica tradicional, como los congresos científicos o conferencias que ocurren en aulas o auditorios de las universidades. Los espacios ideales para eventos presenciales podrían ser espacios públicos de una ciudad, como parques, plazas, cafeterías, en los cuales el espacio invite a la conversación y la interacción social (Selin et al., 2017). 


\subsection{Diseñar el enfoque para el contenido y mensajes científicos}

En la comunicación estratégica el enfoque del mensaje para desarrollar contenido sobre diversos temas apela a los esquemas mentales-interpretativos de las audiencias, según su cultura, edad y entorno. Dichos esquemas permiten que las audiencias comprendan la información que reciben (Entman, 1993). Por ejemplo, para desarrollar una campaña de comunicación se desarrollan estudios de mercado sobre el perfil de la audiencia, para conocer de mejor forma sus características para desarrollar un concepto comunicacional (Bubela et al., 2009). Sin embargo, en la comunicación científica la tarea de definir un enfoque para el contenido y los mensajes para los hallazgos de un proyecto de investigación es distinta.

Conforme a los resultados de este estudio, ambos casos permitieron confirmar mediante el diálogo participativo de los cuatro talleres, una estrategia para identificar el tipo de contenido que es interesante para las audiencias y diseñar mensajes. La guía teórica de la teoría del framing, aplicada a la comunicación científica, establece que la construcción de un esquema mental debe elaborarse mediante datos de investigación formativa sobre las audiencias, donde se identifiquen situaciones y contextos comunes para las audiencias meta y combinarlos con hallazgos e información científica (White, 2013).

Mediante la guía teórica presentada en la revisión literaria y el esquema de análisis este estudio combinó la investigación formativa de cada caso de estudio, y por otra parte pidió a los investigadores que identifiquen los hallazgos claves de sus proyectos.

Primero, dichos hallazgos fueron presentados y discutidos colaborativamente con los participantes de este estudio. Segundo, en torno a los hallazgos la discusión el análisis se centró en comparar y encontrar distintas anécdotas o espacios de su vida cotidiana en los cuales se podría reflejar los hallazgos de investigación. Este procedimiento permitió identificar situaciones cotidianas para las audiencias, por ejemplo: el refrigerio, el momento del recreo como espacio de juego y diversión, los fines de semana en familia y los juegos entre padres, hijos y hermanos, el juego con las mascotas, y cocinar como actividad divertida con amigos o familia. De estas situaciones, se elaboraron bocetos de ejemplos de publicaciones para redes sociales, la sugerencia de crear recursos online de rutinas de actividad física, recetas de cocina, y formatos para eventos informales.

\subsection{Selección de canales y formatos de comunicación}

Poca investigación se ha realizado sobre los canales de comunicación adecuados para la comunicación científica. Sin embargo, los parámetros de la teoría del médium (Meyrowitz, 2009) han permitido explorar las características de los medios masivos, y los nuevos medios 2.0 e identificar conforme al contenido, si ciertos medios son mejores para comunicar cierta información para el entendimiento de los públicos. Por ejemplo: si la prensa escrita es mejor para comunicar hallazgos sobre nutrición, pero en cambio los medios audiovisuales permiten educar y motivar a las audiencias sobre la actividad física.

En el contexto de la comunicación científica, como se observa en estudios previos, la necesidad de facilitar el diálogo es prioritario. Actualmente, las redes sociales ofrecen estos espacios de comunicación inmediata, pero con ciertas características técnicas, 
como los nuevos componentes audiovisuales predominantes y las transmisiones en vivo (Instagram TV, Facebook Live,). Dichas características podrían ser aprovechadas para la comunicación científica (Collins, Shiffman \& Rock, 2016).

En este estudio, participantes e investigadores concluyeron que incorporar canales de redes sociales sería beneficioso para motivar el diálogo y la participación de investigadores con sus audiencias meta, además de ofrecer recursos útiles para incorporar hábitos saludables a su rutina diaria. Estudios existentes evidencian que los científicos que usan las redes sociales para comunicar su trabajo han logrado mayor notoriedad en la opinión pública y los medios de comunicación (Pearce et al., 2015). De igual manera, compartir sus publicaciones científicas en Twitter ha contribuido a darle mayor impacto científico a su trabajo, con mayor número de citaciones, y la oportunidad de colaborar con otros expertos (Liang et al., 2014).

\subsection{Implicaciones clave para la comunicación científica en las américas}

De igual manera, es importante clarificar, que este estudio se refiere y limita a dos casos de estudio específicos en el área de programas de salud preventiva infantil, por lo cual las sugerencias y conclusiones no podrán ser generalizadas en su totalidad, pero sí podrán ser empleadas como guías generales para la comunicación científica.

Implementar comunicación científica que promueva el diálogo ciencia-sociedad, es hoy en día una necesidad latente. A continuación, se presentan algunas consideraciones que los investigadores y los comunicadores estratégicos deben tener en cuenta al trabajar con programas de salud en Estados Unidos y Ecuador.

Primero, el esfuerzo a nivel profesional y de recursos financieros que implica planificar, implementar y evaluar iniciativas de comunicación científica para los investigadores. Adicionalmente, la falta de formación en esta disciplina evidencia que generalmente los investigadores en su formación académica, no cuentan con entrenamiento y preparación en comunicación científica (Peters, 2013). En el caso de los profesionales de la comunicación organizacional y estratégica, es similar ya que la comunicación científica rara vez es parte de la formación de los profesionales de la comunicación.

De igual forma, en el escenario práctico de la investigación tanto en Estados Unidos como en Ecuador, los investigadores no tienen motivación y/o obligación de comunicar a la sociedad los resultados de sus investigaciones. Los parámetros de promoción de docentes-investigadores se limitan a la acumulación de publicaciones científicas (Da Cruz, 2018). Sin embargo, en el caso de Ecuador, los requerimientos de vinculación con la sociedad, podrían ser alternativas para implementar actividades de comunicación científica desde las universidades.

Por otra parte, tanto en Estados Unidos y Ecuador, los fondos de investigación de universidades y organizaciones científicas públicas y privadas, no ofrecen oportunidades de fondos para el desarrollo de la comunicación científica hacia la sociedad (Massarani et al., 2016). La comunicación científica, a pesar de ser una iniciativa generalmente bien percibida y reconocida como necesaria, se limita a la voluntad de los investigadores, y ninguna política que motive su desarrollo.

Sin embargo, una oportunidad para la comunicación científica son también las iniciativas de ciencia ciudadana (Cooper, 2016). Mediante el esquema propuesto por la ciencia ciudadana, los investigadores podrían diseñar estudios colaborativos con 
personas de diversa edad y nivel de educación, que se enfoquen en la participación colaborativa y beneficio abierto de hallazgos de investigación. En este proceso, la comunicación científica tendrá un rol estratégico en la facilitación de tácticas que promuevan el diálogo ciencia-sociedad.

\section{Bibliografía}

Abbott, E., Corbin, L., \& Neibergall, J. (2000). Computer adoption levels of Iowa dailies and weeklies. Newspaper Research Journal, 21(2), pp. 84-94.

Andrade, S., Lachat, C., Ochoa-Aviles, A., Verstraeten, R., Huybregts, L., Roberfroid, D., \& Cardon, G. (2014). A school-based intervention improves physical fitness in Ecuadorian adolescents: A cluster-randomized controlled trial. International Journal of Behavioral Nutrition and Physical Activity, 11(1), pp. 153-170.

Arroyave, J. (2006, June). The emergence of diffusion theory in Latin America: A critical analysis. Paper presented at the annual meeting of the International Communication Association. Dresden, Germany: International Communication Association.

Beltrán, S. (1976). Investigación en comunicación en Latinoamérica: Indagación con anteojeras]. Paper presented at the annual meeting of the International Scientific Conference on Mass Communication and Social Consciousness in a Changing World. Leipzig, Germany.

Bordenave, J. D. (1976). Communication of agricultural innovations in Latin America: The need for new models. Communication Research, 3(2), pp. 135-154.

Brossard, D., Lewenstein, B., \& Bonney, R. (2005). Scientific knowledge and attitude change: The impact of a citizen science project. International Journal of Science Education, 27(9), pp. 1099-1121.

Brossard, D., \& Scheufele, D. A. (2013). Science, new media, and the public. Science, 339(6115), pp. $40-41$.

Bowater, L., \& Yeoman, K. (2013). Science communication: A practical guide for scientists. London, UK: Wiley \& Blackwell.

Bubela, T., Nisbet, M. C., Borchelt, R., Brunger, F., Critchley, C., Einsiedel, E., ... \& Jandciu, E. W. (2009). Science communication reconsidered. Nature Biotechnology, 27(6), pp. 514-518.

Bucchi, M. (2008). Of deficits, deviations, and dialogues: Theories of public communication of science. In M. Bucchi \& B. Trench (Eds.), Handbook of public communication of science and technology, pp. 57-76. New York, NY: Routledge.

Bucchi, M., \& Trench, B. (Eds.). (2008). Handbook of public communication of science and technology. New York, NY: Routledge.

Collins, K., Shiffman, D., \& Rock, J. (2016). How are scientists using social media in the workplace? PloS One, 11(10), e0162680.

Cooper, C. (2016). Citizen science: How ordinary people are changing the face of discovery. London, UK: Gerald Duckworth \& Co.

Chapin, M., \& Threlkeld, B. (2001). Indigenous landscapes. A Study in Ethnocartography. Arlington, VA: Center for the Support of Native Lands.

Chevalier, J. M., \& Buckles, D. J. (2013). Participatory action research: Theory and methods for engaged inquiry. Thousand Oaks, CA: Routledge.

Crawford, C. B., \& Strohkirch, C. S. (2002). Leadership education for knowledge organizations: A primer. Journal of Leadership Education, 1(2), pp. 18-33.

Da Cruz, C. G. (2018). Community-engaged scholarship: Toward a shared understanding of practice. The Review of Higher Education, 41(2), pp. 147-167.

Dunwoody, S. (2014). Science journalism: Prospects in the digital age. In M. Bucchi \& B. Trench (Eds.), Handbook of public communication of science and technology, pp. 43-55. New York, NY: Routledge. 
Dupagne, M. (1999). Exploring the characteristics of potential high-definition television adopters. Journal of Media Economics, 12(1), pp. 35-50.

Dutton, W. H., Rogers, E. M., \& Jun, S. H. (1987). Diffusion and social impacts of personal computers. Communication Research, 14(2), pp. 219-250.

Entman, R. M. (1993). Framing: Toward clarification of a fractured paradigm. Journal of Communication, 43(4), pp. 51-58.

Flyvbjerg, B. (2001). Making social science matter: Why social inquiry fails and how it can succeed again. Cambridge, UK: Cambridge University Press.

Friedman, S. M., Dunwoody, S., \& Rogers, C. L. (1999). Communicating uncertainty: Media coverage of new and controversial science. New York, NY: Routledge.

Gamson, W. (1992). Talking politics. New York, NY: Cambridge University Press.

Glaser, B. G., \& Strauss, A. L. (1967). The discovery of grounded theory: Strategies for qualitative research. Chicago, IL: Aldire.

Gregory, J., \& Miller, S. (1998). Science in public: Communication, culture, and credibility. Cambridge, MA: Plenum.

Grier, S., \& Bryant, C. A. (2005). Social marketing in public health. Annual Review of Public Health, 26, 319-339.

Guzzetti, B. J., \& Gamboa, M. (2004). Zines for social justice: Adolescent girls writing on their own. Reading Research Quarterly, 39(4), pp. 408-436.

Herriott, R. E., \& Firestone, W. A. (1983). Multisite qualitative policy research: Optimizing description and generalizability. Educational Researcher, 12(2), pp. 14-19.

Holliman, R., Collins, T., Jensen, E., \& Taylor, P. (2009). ISOTOPE: Informing science outreach and public engagement. Final report of the NESTA-funded ISOTOPE project. Milton Keynes, UK: Open University.

Jensen, E., \& Holliman, R. (2009). Investigating science communication to inform science outreach and public engagement, pp. 55-71. Oxford, UK: Oxford University Press.

Katz, E. (1957). The two-step flow of communication: An up-to-date report on an hypothesis. Public Opinion Quarterly, 21(1), pp. 61-78

Kesby, M. (2000). Participatory diagramming: Deploying qualitative methods through an action research epistemology. Area, 32(4), pp. 423-435.

Leach, J., Yates, S., \& Scanlon, E. (2008). Models of science communication. In Holliman, R., Whitelegg, E., Scanlon, E., Smidt, S., \& Thomas, J. (Eds.), Investigating science communication in the information age, pp. 128-146. London, UK: Oxford University Press.

Liang, X., Su, L. Y.F., Yeo, S. K., Scheufele, D. A., Brossard, D., Xenos, M., \& Corley, E. A. (2014). Building buzz: Scientists communicating science in new media environments. Journalism $\mathcal{E}$ Mass Communication Quarterly, 91(4), pp. 772-791.

Lin, C. A. (1998). Exploring personal computer adoption dynamics. Journal of Broadcasting $\mathcal{E}$ Electronic Media, 42(1), pp. 95-112.

Listerman, T. (2010). Framing of science issues in opinion-leading news: International comparison of biotechnology issue coverage. Public Understanding of Science, 19(1), pp. 5-15.

Marshall, C., \& Rossman, G. B. (2014). Designing qualitative research. Thousand Oaks, CA: Sage.

Martín-Barbero, J. (1993). Communication, culture, and hegemony: From the media to mediations. London, UK: Sage.

Massarani, L., Reynoso-Haynes, E., Murriello, S., \& Castillo, A. (2016). Posgrado en comunicación de la Ciencia en América Latina: Un mapa y algunas reflexiones. [Science Communication Postgraduate Studies in Latin America: A map and some food for thought]. JCOM, 15(05), A03.

McNiff, J. (2014). Writing and doing action research. London, UK: Sage.

Meyrowitz, J. (2009). Medium theory. In D. Crowley \& D. Mitchell (Eds.), Communication theory today, pp. 50-77. Redwood City, CA: Stanford University Press.

Pearce, W., Brown, B., Nerlich, B., \& Koteyko, N. (2015). Communicating climate change: Conduits, content, and consensus. Wiley Interdisciplinary Reviews: Climate Change, 6(6), pp. 613-626. 
Peters, H. P. (2013). Gap between science and media revisited: Scientists as public communicators. Proceedings of the National Academy of Sciences, 110 (Suppl. 3), pp. 14102-14109.

Rocha, M., Massarani, L., \& Pedersoli, C. (2017). La divulgación de la ciencia en América Latina: Términos, definiciones y campo académico. En L. Massarani, M. Rocha, C. Pedersoli, C. Almeida, L. Amorim, M. Cambre, A.C. Nepote, N. Rocha, J. Aguirre, J.C. Goncalvez, L. Cordioli \& F. Ferreira (Eds.), Aproximaciones a la investigación en divulgación de la ciencia en América Latina a partir de sus artículos académicos, pp. 39-58. Rio de Janeiro, Brasil: Fiocruz-COC.

Rogers, E. M. (2010). Diffusion of innovations. New York, NY: Simon and Schuster.

Selin, C., Rawlings, K. C., de Ridder-Vignone, K., Sadowski, J., Altamirano Allende, C., Gano, G., \& Guston, D. H. (2017). Experiments in engagement: Designing public engagement with science and technology

Stilgoe, J., Lock, S. J., \& Wilsdon, J. (2014). Why should we promote public engagement with science? Public Understanding of Science, 23(1), pp. 4-15.

Weigold, M. F. (2001). Communicating science: A review of the literature. Science communication, 23(2), pp. 164-193.

White, J. M. (2013). Translating science, health and technology: Reporters as knowledge transfer intermediaries. Online Journal of Communication and Media Technologies, 3(2), pp. 227.

Wynne, B. (1995). Public understanding of science. In S. Jasanoff, G. E. Markle, J. C. Peterson, \& T. Pinch (Eds.), Handbook of science and technology studies, pp. 361-388. Thousand Oaks, CA: Sage.

Wong, N. T., Zimmerman, M. A., \& Parker, E. A. (2010). A typology of youth participation and empowerment for child and adolescent health promotion. American Journal of Community Psychology, 46(1-2), pp. 100-114.

Yin, R. K. (2017). Case study research and applications: Design and methods. (6th ed.). Thousand Oaks, CA: Sage. 